\title{
Diferencias de género en el empleo TIC*
}

\author{
Carlos Iglesias Fernández ${ }^{\mathrm{a}}$
}

Universidad de Alcalá e Instituto de Análisis Económico y Social (IAES)

\section{Raquel Llorente Heras ${ }^{\mathrm{b}}$}

Universidad Autónoma de Madrid e Instituto de Análisis Económico y Social (IAES)

\section{Diego Dueñas Fernández ${ }^{c}$}

Universidad de Alcalá e Instituto de Análisis Económico y Social (IAES)

\section{RESUMEN}

La fuerte expansión que han experimentado las nuevas tecnologías en España en los últimos años ha propiciado la creación de nuevos nichos de empleo a los que la parte feme-

* Este artículo forma parte del proyecto «SOCIEDAD DE LA INFORMACIÓN EN IGUALDAD Y PARA LA IGUALDAD: Desajuste entre participación y posición de las mujeres en la investigación y el empleo TIC», dirigido por Internet Interdisciplinary, Universitat Oberta de Catalunya, a la cual lo autores agradecen la oportunidad que les ha brindado a través de la colaboración en dicho proyecto.

${ }^{a}$ Autor para correspondencia: Raquel Llorente Heras. Facultad de CC Económicas y Empresariales, Campus de Cantoblanco, 28049 Madrid. Teléfono: +34 91 4972961, Fax: +34 914976930, Correo electrónico: raquel.llorente@uam.es.

b Carlos Iglesias Fernández. Departamento de Economía Aplicada. Universidad de Alcalá. Plaza de la Victoria, 128802 Alcalá de Henares. Teléfono: +34 9188552 25. Correo electrónico: carlos.iglesias@uah.es

c Diego Dueñas Fernández. Departamento de Fundamentos e Historia Económica. Universidad de Alcalá. Plaza de la Victoria, 128802 Alcalá de Henares. Teléfono: +34 918855236. correo electrónico: diego.duenas@uah.es.

Recibido: junio 2009 / Aceptado: diciembre 2009. 
nina de la población ha acudido de forma considerable, sobre todo si lo comparamos con el incremento de la ocupación masculina en los mismos empleos. A partir de esta evidencia empírica, nos planteamos en este artículo si el empleo TIC presenta mejores resultados a la hora de medir la segregación por género que gobierna el mercado de trabajo español, donde las mujeres se ven seriamente perjudicadas. Los datos nos dicen que, si bien dentro del empleo TIC se produce mayor igualdad entre sexos, no podemos concluir de forma definitiva que no sigan existiendo disparidades por razón de género. A la luz de estos resultados y ya que hombres y mujeres no se relacionan de la misma forma con el empleo TIC, se realizan estudios econométricos para determinar cómo afectan determinadas variables personales y laborales a la probabilidad de trabajar en el ámbito de las TIC.

Palabras claves: empleo TIC, segregación, género, desigualdad.

Códigos JEL: J16, J70, O33,

\begin{abstract}
The strong growth new technologies have experienced in Spain in recent years has caused the creation of new employment niches for which female population has gone considerably, specially if we compare it with the increase of men employed in the same jobs. From this evidence, we propose in this paper if ICT employment shows better results when measuring gender discrimination in Spanish labour market, where women are seriously injured. Data show that while ICT employment is more equality, we ca not conclude definitively that there are still no disparities based on gender.
\end{abstract}

Keywords: ICT employment, gender, inequality

JEL Classification: J70, O33,

\title{
1. INTRODUCCIÓN Y DEFINICIÓN DE CONCEPTOS
}

A lo largo del siglo xx las economías occidentales han experimentado distintos procesos de transición, evolucionando desde una sociedad industrial (acceso masivo a bienes producidos por otros), pasando por una sociedad postindustrial (acceso masivo a servicios producidos por otros), y de ésta, a lo que se ha denominado Sociedad de la Información y Nueva Economía, la cual ha supuesto una auténtica revolución tanto para nuestras vidas como para los sistemas económicos en los que estamos inmersos (Castells, 2000). Este nuevo escenario se ha cimentado a partir de los avances tecnológicos experimenta- 
dos a nivel mundial en las últimas décadas, integrando sinérgicamente la informática, la microelectrónica y las telecomunicaciones, así como el uso de nuevos desarrollos, especialmente Internet, el cual ha modificado sustancialmente las relaciones entre los agentes económicos. Dado su carácter de sistema tecnológico (Freeman, Clarck y Soete, 1985), las implicaciones que se derivan de su uso y difusión revisten en la práctica un carácter de generalidad (Freeman y Soete, 1996), presentando una especial trascendencia en el ámbito social y laboral (Ducatel, 1994), ya que dicha tecnología requiere de trabajadores cualificados en su utilización, por lo que una batería de conceptos, entre los que destacan la educación, la cualificación o el conocimiento, entre otros, surgen como indispensables a la hora de constituir el entramado laboral en las empresas (Castaño y cols., 2005).

Por otra parte, en nuestro país, el mercado de trabajo ha estado protagonizado en las últimas décadas por varios acontecimientos que han supuesto la activación del desarrollo económico y social que actualmente disfrutamos, de entre los cuales, la fuerte incorporación de la mujer al mundo laboral es, sin duda alguna, el más destacable, ya que ha permitido que hombres y mujeres formen parte de la fuerza laboral de nuestro país, alterando en sentido positivo, como así reconoce la literatura elaborada al respecto (Castaño y cols., 2003; Iglesias, 2005), numerosos estereotipos sociales y económicos establecidos hasta entonces.

Sin embargo, aun quedan pendientes algunas cuestiones importantes que no han quedado resueltas en este proceso, como son, entre otras, la igualdad por razón de género en el mercado laboral y la ausencia de discriminación social y económica que sufren las mujeres en sus respectivos trabajos y que desembocan, en numerosas ocasiones, en peores condiciones laborales y en menores salarios que los hombres en los mismos empleos (De la Rica, 2007). La desigualdad de la que aquí hablamos queda patente si analizamos la evolución que los índices de segregación han experimentado en los últimos años. Si bien este fenómeno no ha sido ampliamente estudiado para el caso del mercado laboral español, principalmente en lo que se refiere a las causas que lo originan, podemos concluir a la luz de diversos trabajos (Cebrian y Moreno, 2008; Maté et al, 2002) que dichos índices han experimentado un incremento si consideramos el mercado laboral en su conjunto, y que dicho mercado presenta una profunda diferenciación de género, tanto si lo analizamos desde el punto de vista de los sectores de actividad como si lo hacemos desde el punto de vista de las ocupaciones laborales (Iglesias y Llorente, 2009).

La importancia de este trabajo radica en comprobar si esta lacra social que arrastra nuestro mercado laboral se encuentra también presente en el empleo asociado a las Tecnologías de la Información y Comunicación (TIC, en adelante), ya que, al menos desde un punto de vista intuitivo, se trata de un sector en el que debe predominar cierta paridad social, no sólo en cuanto al género sino también en lo que se refiere a la capacidad y a la cualificación de los trabajadores. 
Para determinar qué se entiende por empleo TIC, y, por tanto, las actividades y ocupaciones TIC, el punto de partida no debe ser otro que las directrices marcadas por la Organización para el Desarrollo y la Cooperación Económica (OCDE, en adelante), y que definen el empleo TIC como aquella parte del empleo que guarda una especial relación con las TICs (OCDE, 2004). Para su medición, la OCDE utiliza dos aproximaciones distintas. De acuerdo con la primera, se entiende por empleo TIC aquel ubicado en el sector productor de bienes y servicios TIC (sector TIC); desde la segunda de las perspectivas, el empleo TIC es todo aquel empleo cuyas características cualificativas muestran una especial relación con las TIC (empleo con cualificaciones orientadas a las TIC o ICT-skilled employment).

A efectos operativos, la mayor parte de la investigación, se centrará en el estudio de las ocupaciones que se relacionan con las TICs por lo que las ocupaciones consideradas de acuerdo a la CNO-94 son un total de 32, las cuales se presentan en el Anexo 1. Las ocupaciones laborales restantes las consideraremos como ocupaciones no TIC.

A partir de esta definición, nuestro objetivo radica en analizar cómo han evolucionado los indicadores laborales que nos proporcionen información en cuanto a la posición laboral de la mujer, y que nos permitan comparar dicha información con los indicadores por género que nos ofrece el mercado de trabajo español en su conjunto. De esta forma, lo que aquí se propone consiste en contrastar una doble hipótesis:

- Por un lado, si la evolución de la población femenina dentro del empleo TIC es similar a la evolución de la mujer en el empleo no TIC, o si, por el contrario, se están produciendo comportamientos divergentes significativos.

- Por otro, si la población femenina dentro de las TICs tiene pautas de comportamiento similares a las del género opuesto, es decir, si el empleo TIC es un sector en el que la diferenciación por razón de género desaparece.

Para ello, el artículo queda estructurado de la siguiente manera. En el apartado 2 se lleva a cabo un análisis descriptivo de la situación laboral de hombres y mujeres en el empleo TIC, desde una perspectiva vertical, es decir, por ocupaciones. El apartado 3 aborda un análisis similar desde una perspectiva horizontal, es decir, por sectores de actividad, aunque siempre teniendo en cuenta la diferenciación entre ocupaciones TIC y no TIC. En el cuarto apartado se llevará a cabo un profundo análisis para determinar cuáles son las características laborales y personales que determinan las diferencias por género dentro del empleo TIC. Para finalizar, en el apartado 5 se resaltan las principales conclusiones. Los datos han sido extraidos de la Encuesta de Población Activa (EPA, en adelante) desagregada a 3 dígitos (lo cual aporta mayor interés por el nivel de detalle con el que se analiza el mercado laboral), tomando como referencia los segundos trimestres de los años 2002 y 2007. 


\section{EMPLEO, TIC Y GÉNERO: UNA PERSPECTIVA VERTICAL}

En primer lugar, vamos a analizar el empleo TIC y no TIC desde una perspectiva vertical, es decir, considerando la forma en que hombres y mujeres se distribuyen en la estructura de ocupaciones laborales. Estos datos se presentan en el cuadro 1. A la luz de los datos expuestos, la primera impresión de la situación por género en el empleo TIC nos induce a pensar que la mujer muestra una mayor relación con este tipo de empleo ya que en ambos años presenta mayores porcentajes de empleo que los hombres en las ocupaciones relacionadas con la tecnología, información y comunicación.

CUADRo 1.-Número de ocupados para el total de la población, hombres y mujeres, y su distribución en las Ocupaciones TIC y no TIC, para el año 2002 y 2007

\begin{tabular}{|l|c|c|c|}
\cline { 2 - 4 } \multicolumn{1}{c|}{} & \multicolumn{3}{c|}{2002} \\
\cline { 2 - 4 } \multicolumn{1}{c|}{} & ESPAÑA & HOMBRES & MUJERES \\
\hline Ocupados (en mill.) & 16,5 & 10,3 & 6,2 \\
\hline Ocupaciones TIC & $20,34 \%$ & $18,84 \%$ & $22,82 \%$ \\
\hline Ocupaciones no TIC & $79,66 \%$ & $81,16 \%$ & $77,29 \%$ \\
\hline \multirow{2yyy}{*}{} & \multicolumn{3}{c|}{2007} \\
\hline Ocupados (en mill.) & ESPAÑA & HOMBRES & MUJERES \\
\hline Ocupaciones TIC & 20,3 & 11,9 & 8,4 \\
\hline Ocupaciones no TIC & $78,29 \%$ & $19,80 \%$ & $23,42 \%$ \\
\hline
\end{tabular}

CUADro 2.--Índices de participación masculina y femenina en las ocupaciones TIC y no TIC en el año 2002 y 2007

\begin{tabular}{|l|c|c|}
\cline { 2 - 3 } \multicolumn{1}{c|}{} & \multicolumn{2}{c|}{ Ocupaciones TIC } \\
\cline { 2 - 3 } \multicolumn{1}{c|}{} & 2002 & 2007 \\
\hline HOMBRES & $57,63 \%$ & $54,71 \%$ \\
\hline MUJERES & $42,37 \%$ & $45,29 \%$ \\
\hline \multirow{2}{*}{\multicolumn{1}{c|}{}} & \multicolumn{2}{c|}{ Ocupaciones no TIC } \\
\cline { 2 - 3 } \multicolumn{1}{c|}{2002} & 2007 \\
\hline HOMBRES & $63,60 \%$ & $60,10 \%$ \\
\hline
\end{tabular}


El cuadro 1 se completa con el cuadro 2, que recoge la participación masculina y femenina ${ }^{1}$, tanto en las ocupaciones TIC como en las ocupaciones no TIC. Podemos extraer algunas conclusiones importantes a partir de los datos presentados en el cuadro anterior:

- Los hombres presentan un mayor peso que las mujeres en ambos tipos de ocupaciones, tanto para el 2002 como para el 2007, aunque es mayor en el empleo no TIC.

- Al contrario, el porcentaje de mujeres en las ocupaciones referidas a las TIC es mayor que el porcentaje en las no TIC.

— La brecha de género referida a la participación es más reducida en el empleo TIC.

- Tanto en las ocupaciones TIC como en las ocupaciones no TIC la evolución de los porcentajes ha resultado favorable a la mujer, que ve aumentar sus ponderaciones en detrimento de la de los hombres.

Otra perspectiva interesante consiste en calcular las pautas de concentración de mujeres y hombres en ambos tipos de empleo. Este indicador nos informará sobre las ubicaciones más frecuentes dentro del empleo masculino y femenino. En los cuadros 3 y 4 presentamos los datos para el empleo TIC y no TIC sobre dicha concentración.

CuAdro 3.-Índices de concentración para el total de la población, hombres y mujeres en el año 2002 y 2007 , para las ocupaciones TIC

\begin{tabular}{|c|c|c|c|c|c|}
\hline \multicolumn{2}{|c|}{2002} \\
\hline ESPAÑA & \multicolumn{2}{|c|}{ HOMBRES } & \multicolumn{2}{c|}{ MUJERES } \\
\hline $\begin{array}{c}\text { Profesionales de apoyo a la } \\
\text { gestión administrativa }\end{array}$ & $17,33 \%$ & $\begin{array}{c}\text { Representantes de comercio } \\
\text { y técnicos de venta }\end{array}$ & $14,37 \%$ & $\begin{array}{c}\text { Profesionales de apoyo a la } \\
\text { gestión administrativa }\end{array}$ & $26,32 \%$ \\
\hline $\begin{array}{c}\text { Auxiliares administrativos } \\
\text { sin tareas de atención al } \\
\text { público }\end{array}$ & $13,39 \%$ & $\begin{array}{c}\text { Profesionales de apoyo a la } \\
\text { gestión administrativa }\end{array}$ & $10,72 \%$ & $\begin{array}{c}\text { Auxiliares administrativos } \\
\text { con tareas de atención al } \\
\text { público }\end{array}$ & $20,85 \%$ \\
\hline $\begin{array}{c}\text { Auxiliares administrativos } \\
\text { con tareas de atención al } \\
\text { público }\end{array}$ & $12,28 \%$ & $\begin{array}{c}\text { Mecánicos y ajustadores } \\
\text { de equipos elécticos y } \\
\text { electrónicos }\end{array}$ & $9,26 \%$ & $\begin{array}{c}\text { Auxiliares administrativos } \\
\text { sin tareas de atención al } \\
\text { público }\end{array}$ & $20,85 \%$ \\
\hline $\begin{array}{c}\text { Las 5 ocupaciones con } \\
\text { mayor concetración }\end{array}$ & $58,86 \%$ & $\begin{array}{c}\text { Las 5 ocupaciones con } \\
\text { mayor concetración }\end{array}$ & $48,25 \%$ & $\begin{array}{c}\text { Las 5 ocupaciones con } \\
\text { mayor concetración }\end{array}$ & $77,42 \%$ \\
\hline
\end{tabular}

1 Tanto por ciento de mujeres y hombres sobre el total del empleo TIC y no TIC. 
Cuadro 3 (cont.).- Índices de concentración para el total de la población, hombres y mujeres en el año 2002 y 2007, para las ocupaciones TIC

\begin{tabular}{|c|c|c|c|c|c|}
\hline \multicolumn{2}{|c|}{2007} & \multicolumn{2}{c|}{ HOMBRES } & \multicolumn{2}{c|}{ MUJERES } \\
\hline $\begin{array}{c}\text { ESPAÑA } \\
\text { Profesionales de apoyo a la } \\
\text { gestión administrativa }\end{array}$ & $18,01 \%$ & $\begin{array}{c}\text { Representantes de comercio } \\
\text { y técnicos de venta }\end{array}$ & $14,62 \%$ & $\begin{array}{c}\text { Profesionales de apoyo a la } \\
\text { gestión administrativa }\end{array}$ & $27,02 \%$ \\
\hline $\begin{array}{c}\text { Auxiliares administrativos } \\
\text { con tareas de atención al } \\
\text { público }\end{array}$ & $12,67 \%$ & $\begin{array}{c}\text { Profesionales de apoyo a la } \\
\text { gestión administrativa }\end{array}$ & $10,55 \%$ & $\begin{array}{c}\text { Auxiliares administrativos } \\
\text { con tareas de atención al } \\
\text { público }\end{array}$ & $20,95 \%$ \\
\hline $\begin{array}{c}\text { Representantes de comercio } \\
\text { y técnicos de venta }\end{array}$ & $9,86 \%$ & $\begin{array}{c}\text { Mecánicos y ajustadores } \\
\text { de equipos elécticos y } \\
\text { electrónicos }\end{array}$ & $7,97 \%$ & $\begin{array}{c}\text { Auxiliares administrativos } \\
\text { sin tareas de atención al } \\
\text { público }\end{array}$ & $15,83 \%$ \\
\hline $\begin{array}{c}\text { Las 5 ocupaciones con } \\
\text { mayor concetración }\end{array}$ & $56,00 \%$ & $\begin{array}{c}\text { Las } 5 \text { ocupaciones con } \\
\text { mayor concetración }\end{array}$ & $45,37 \%$ & $\begin{array}{c}\text { Las 5 ocupaciones con } \\
\text { mayor concetración }\end{array}$ & $73,68 \%$ \\
\hline
\end{tabular}

La comparación por género nos invita a concluir que existe, claramente, mayor concentración en las ocupaciones TIC para la población femenina que para la masculina, tanto en el 2002 como en el 2007, produciéndose una ligera reducción de la concentración en ambos colectivos. Igualmente podemos decir que, en términos generales, la mujer tiene una ocupación TIC que se relaciona muy directamente con la labor administrativa mientras que el perfil masculino se encuentra más diversificado entre el comercio, la gestión administrativa y la mecánica.

CUADRo 4.--Índices de concentración para el total de la población, hombres y mujeres en el año 2002 y 2007 , para las ocupaciones no TIC

\begin{tabular}{|c|c|c|c|c|c|}
\hline \multicolumn{2}{|c|}{ ESPAÑA } & \multicolumn{2}{c|}{ HOMBRES } & \multicolumn{2}{c|}{ MUJERES } \\
\hline $\begin{array}{c}\text { Dependientes en tiendas, } \\
\text { almacenes, quioscos y } \\
\text { mercados }\end{array}$ & $5,77 \%$ & Albañiles y mamposteros & $6,58 \%$ & $\begin{array}{c}\text { Dependientes en tiendas, } \\
\text { almacenes, quioscos y } \\
\text { mercados }\end{array}$ & $10,97 \%$ \\
\hline $\begin{array}{c}\text { Personal de limpieza de } \\
\text { oficinas, hoteles y similares }\end{array}$ & $4,47 \%$ & Conductores de camiones & $4,23 \%$ & $\begin{array}{c}\text { Personal de limpieza de } \\
\text { oficinas, hoteles y similares }\end{array}$ & $10,84 \%$ \\
\hline Albañiles y mamposteros & $4,20 \%$ & Peones de la construcción & $4,08 \%$ & Empleadas del hogar & $6,82 \%$ \\
\hline $\begin{array}{c}\text { Las 5 ocupaciones con } \\
\text { mayor concetración }\end{array}$ & $20,28 \%$ & $\begin{array}{c}\text { Las 5 ocupaciones con } \\
\text { mayor concetración }\end{array}$ & $21,52 \%$ & $\begin{array}{c}\text { Las 5 ocupaciones con } \\
\text { mayor concetración }\end{array}$ & $36,87 \%$ \\
\hline
\end{tabular}


CUADRo 4 (cont.).- - Índices de concentración para el total de la población, hombres y mujeres en el año 2002 y 2007, para las ocupaciones no TIC

\begin{tabular}{|c|c|c|c|c|c|}
\hline \multicolumn{2}{|c|}{} & \multicolumn{2}{c|}{ HOMBRES } & \multicolumn{2}{c|}{ MUJERES } \\
\hline $\begin{array}{c}\text { Dependientes en tiendas, } \\
\text { almacenes, quioscos y } \\
\text { mercados }\end{array}$ & $5,65 \%$ & Albañiles y mamposteros & $7,22 \%$ & $\begin{array}{c}\text { Personal de limpieza de } \\
\text { oficinas, hoteles y similares }\end{array}$ & $10,78 \%$ \\
\hline $\begin{array}{c}\text { Personal de limpieza de } \\
\text { oficinas, hoteles y similares }\end{array}$ & $4,82 \%$ & Peones de la construcción & $4,97 \%$ & $\begin{array}{c}\text { Dependientes en tiendas, } \\
\text { almacenes, quioscos y } \\
\text { mercados }\end{array}$ & $10,33 \%$ \\
\hline Albañiles y mamposteros & $4,35 \%$ & Conductores de camiones & $4,25 \%$ & Empleadas del hogar & $8,90 \%$ \\
\hline $\begin{array}{c}\text { Las 5 ocupaciones con } \\
\text { mayor concetración }\end{array}$ & $21,87 \%$ & $\begin{array}{c}\text { Las 5 ocupaciones con } \\
\text { mayor concetración }\end{array}$ & $22,72 \%$ & $\begin{array}{c}\text { Las 5 ocupaciones con } \\
\text { mayor concetración }\end{array}$ & $38,71 \%$ \\
\hline
\end{tabular}

Por tanto, en el caso de las ocupaciones que no están relacionadas con las TIC también se observa una mayor concentración para la población femenina que para la masculina, tanto en el 2002 como en el 2007, produciéndose un ligero aumento de la concentración en ambos colectivos. Igualmente podemos decir que, en términos generales, la mujer tiene una ocupación no TIC que está relacionada con la limpieza y la atención al público en mercados y quioscos, mientras que el perfil masculino se encuentra muy concentrado en tareas relacionadas con la construcción y el transporte.

Para profundizar en el análisis, se han calculado dos índices de segregación: el Index of Dissimilarity (ID) propuesto por Duncan y Duncan (Duncan y Duncan, 1955), y el Índice de Karmel y Maclachlan (IP), definido por estos autores en 1988 (Karmel y Maclachlan, 1988). Ambos indicadores son los más utilizados en la literatura encargada de analizar la segregación laboral por género, principalmente para el mercado de trabajo español (Otero y Gradín, 2001; Maté y cols., 2002; Sánchez, 1993, entre muchos otros). El objetivo de plantear el análisis de la segregación mediante la utilización de ambos índices está basado en resolver algunos problemas que plantea el ID, tanto en su medición como en su interpretación ${ }^{2}$, lo cual se resuelve mediante la utilización del iP, que es más adecuado que otros índices planteados en la literatura (Salas, 2004).

El primero de ellos representa el porcentaje de hombres (o de mujeres) que deberían cambiar de ocupación laboral, sin reposición, para que la distribución del empleo en ambos sexos fuese idéntica. Este índice toma valores entre 0 y 1 : un valor nulo para este

2 Véanse, por ejemplo, Watts (1992) y Boisso y cols. (1994). 
índice significa que la distribución de todas las mujeres empleadas entre las diferentes ocupaciones es idéntica a la distribución de los hombres, y un valor igual a la unidad indica que todas las ocupaciones son totalmente masculinas o femeninas. El índice de Duncan y Duncan se define de la siguiente forma:

$$
\mathrm{ID}=\frac{1}{2} \sum\left|\frac{F_{i}}{F}-\frac{M_{i}}{M}\right|
$$

donde $F_{i}$ y $M_{i}$ son el número de mujeres y de hombres en la ocupación $i$, respectivamente; y $F$ y $M$ son el total de mujeres y de hombres empleados, respectivamente.

El segundo índice mide la proporción del empleo total que debería cambiar de ocupación laboral, con reposición, para alcanzar un grado de segregación por sexo nulo, es decir, para que exista la misma proporción de mujeres y hombres en cada ocupación que la que existe a escala agregada. Al mismo tiempo, se mantienen constantes la estructura ocupacional y los porcentajes de participación de cada sexo en el empleo total. Este índice toma valores entre 0 y $1 / 2$ y queda determinado de la siguiente manera:

$$
\mathrm{IP}=\frac{1}{T} \sum\left|(1-a) F_{i}-a M_{i}\right|
$$

donde T es el empleo total y $a$ es la participación de las mujeres en el conjunto del em$\mathrm{pleo}^{3}$. En el siguiente cuadro 5 se presentan los resultados de ambos índices tanto para el empleo TIC como para el empleo no TIC, en 2002 y 2007 :

CuAdro 5.-Evolución de los índices ID e IP para las ocupaciones TIC y no TIC en los años 2002 y 2007 . (Fuente: elaboración propia a partir de la EPA)

\begin{tabular}{|l|c|c|}
\cline { 2 - 3 } \multicolumn{1}{c|}{} & \multicolumn{2}{c|}{ Índice de Duncan y Duncan } \\
\cline { 2 - 3 } \multicolumn{1}{c|}{} & 2002 & 2007 \\
\hline Ocupaciones TIC & 0,4478 & 0,4593 \\
\hline Ocupaciones no TIC & 0,5848 & 0,6088 \\
\hline
\end{tabular}

${ }^{3}$ El índice IP es $2 a(1-a) * I D$, siendo a=F/T. 
Cuadro 5 (cont.).-Evolución de los índices ID e IP para las ocupaciones TIC y no TIC en los años 2002 y 2007. (Fuente: elaboración propia a partir de la EPA)

\begin{tabular}{|l|c|c|}
\hline \multirow{2}{*}{\multicolumn{1}{c|}{}} & \multicolumn{2}{|c|}{ Índice de Karmel y Maclachlan } \\
\cline { 2 - 3 } \multicolumn{1}{c|}{} & 2002 & 2007 \\
\hline Ocupaciones TIC & 0,2187 & 0,2276 \\
\hline Ocupaciones no TIC & 0,3723 & 0,4160 \\
\hline
\end{tabular}

A partir de los datos mostrados, podemos extraer varias conclusiones importantes:

- Para las ocupaciones TIC, y según el ID, en el año 2002 un 44,78 por ciento de hombres (o de mujeres) deberían cambiar de ocupación para que ambos sexos estuvieran igual distribuidos por ocupaciones; en el año 2007 este porcentaje aumentó a 45,93 por ciento. Según el índice IP, en el año 2002, un 21,87 por ciento de los puestos de trabajo deberían pasar de mujeres a hombres (y viceversa) para que el grado de segregación fuera nulo; en el año 2007, este porcentaje había aumentado a 22,76 por ciento.

- En las ocupaciones no TIC, según el ID en el año 2002 un 58,48 por ciento de hombres (o de mujeres) deberían cambiar de ocupación para que ambos sexos estuvieran igual distribuidos por ocupaciones; en el año 2007 este porcentaje aumentó a 60,88 por ciento. Según el índice IP, en el año 2002, un 37,23 por ciento de los puestos de trabajo deberían pasar de mujeres a hombres (y viceversa) para que el grado de segregación fuera nulo; en el año 2007, este porcentaje había aumentado a 41,6 por ciento.

- Por tanto, y según lo anterior, la segregación por razón de género aumenta tanto en las ocupaciones TIC como en las ocupaciones no TIC.

- Si bien el Índice de Duncan y Duncan no permite la comparación entre variables con distinto número de ocupaciones ${ }^{4}$, es decir, comparaciones entre TIC y no TIC, el Índice de Karmel y MacLachlan sí nos permite concluir que la segregación es mucho mayor en el empleo no TIC que en el empleo TIC tanto en el año 2002 como en el 2007, e igualmente poder subrayar un mayor crecimiento en la segregación en las ocupaciones no TIC que en las ocupaciones TIC.

- En definitiva, el empleo TIC muestra un comportamiento mucho más favorable en lo que a igualdad de género se refiere, si bien, este mejor comporta-

4 Para un análisis detallado de las propiedades e interpretación de los índices véase, por ejemplo, Kakwani (1994) o Flückinger y Silber (1999). 
miento no nos permite concluir que la evolución de la segregación dentro de las ocupaciones relacionadas con las TIC sea favorable para la reducción de la discriminación dentro de nuestro mercado laboral.

Para finalizar el análisis vertical vamos a estudiar a través del índice de representación el carácter masculino, femenino o integrado de las ocupaciones TIC. Para ello, siguiendo la línea de otros trabajos (Cáceres y cols., 2004), diremos que la ocupación $i$ está masculinizada (o feminizada) cuando la participación de los hombres (o mujeres) en dicha ocupación $i$ supere en un 25 por ciento a la participación de los hombres (o mujeres) en el total de las ocupaciones. Las ocupaciones que no superaran este umbral no estarán ni masculinizadas ni feminizadas, sino integradas. Los resultados de aplicar este criterio se recogen en los Anexos 2 y 3 .

En el año 2002 eran 17 las ocupaciones TIC que estaban masculinizadas, es decir, un 53,12 por ciento del conjunto de la distribución. En 2007 este número se ha reducido a 14 ocupaciones (desaparecen «Jueces y magistrados», "Consignatarios y agentes en la contratación de mano de obra» y «Mecánicos y ajustadores de equipos eléctricos y electrónicos»), que suponen un 43,75 por ciento del total. En el año 2002 eran 8 las ocupaciones TIC que estaban feminizadas, es decir, un 25 por ciento del total, manteniéndose este porcentaje y las mismas ocupaciones en 2007.

En consecuencia, en el año 2002 ascendían a 7 las ocupaciones TIC que se consideran integradas, es decir, 21,87 por ciento, que eran «Físicos, químicos y asimilados», «Abogados y fiscales», «Otros profesionales del derecho», «Profesionales en administración y dirección de empresa», «Profesionales de apoyo en operaciones financieras y comerciales», «Auxiliares contables y financieros» y «Grabadores de datos». En el año 2007 las ocupaciones TIC integradas pasan a ser 11, un 34,37 por ciento, añadiéndose a las que ya estaban integradas en el 2002 las tres ocupaciones referidas anteriormente que dejaron de estar masculinizadas.

Con respecto a las ocupaciones no TIC, en el año 2002 eran 92 las que estaban masculinizadas, es decir, un 53,49 por ciento, mientras que en 2007 habían aumentado a 98, que representan un 56,98 por ciento del total. En el año 2002 eran 31 las ocupaciones no TIC que estaban feminizadas, es decir, un 18,02 por ciento, incrementándose a 36 en 2007, que suponen el 20,93 por ciento del total. Este balance nos deja un total de 49 ocupaciones no TIC integradas en el año 2002, es decir, un 28,49 por ciento del total, mientras que en el año 2007 habían descendido a 38, que suponen un 19,71 por ciento. De forma sintetizada, la evolución tanto de las ocupaciones TIC como de las ocupaciones no TIC en lo que se refiere al índice de representación se resume en el siguiente gráfico 1 . 
GrÁFICO 1.-Evolución en porcentaje de las ocupaciones TIC y no TIC masculinizadas, feminizadas e integradas en 2002 y 2007

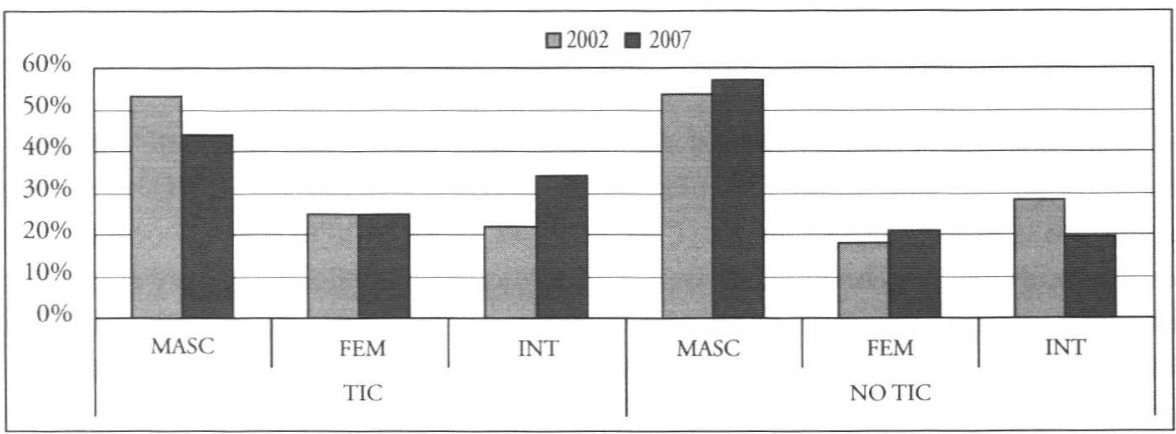

De dicho gráfico podemos extraer las siguientes conclusiones:

- En el empleo TIC se produce una disminución de las ocupaciones masculinizadas, lo cual hace que incrementen las ocupaciones integradas, y no las feminizadas, por lo que podemos decir que, en este sentido, se ha producido un comportamiento a favor de la igualdad de género.

- En el empleo no TIC se produce un incremento de las ocupaciones masculinizadas y de las ocupaciones feminizadas, a costa de las ocupaciones integradas, por lo que, en este caso, existe un comportamiento desfavorable en la búsqueda de la igualdad de género.

- Esta evolución ha producido que en el año 2007 existan claras disparidades entre ambos tipos de empleo en las ocupaciones masculinizadas e integradas.

\section{EMPLEO, TIC Y GÉNERO: UNA PERSPECTIVA HORIZONTAL}

Complementariamente a la anterior perspectiva vertical, vamos ahora a analizar la posición laboral de mujeres y hombres en función de los sectores de actividad en los que se encuentran empleados (análisis horizontal). Nótese que no cambiamos nuestra definición de empleo TIC. Continuamos identificando dentro de este tipo de empleo a todos aquellos trabajadores que desempeñan sus tareas en ocupaciones laborales vinculadas con las TIC. A diferencia del anterior análisis, donde para este colectivo nos fijábamos en la forma en que se distribuían mujeres y hombres a lo largo de la estructura de ocupaciones, ahora nos fijaremos en la forma en que el mismo colectivo se distribuye por sectores de actividad. 
En primer lugar, y de acuerdo con el cuadro número 6, el grupo masculino tiene un mayor peso específico en ambos tipos de actividades, tanto en las TIC como en las no TIC. Igualmente, para el año 2002 y para el año 2007, el porcentaje de hombres en las actividades no TIC es mayor que el porcentaje de hombres en las actividades TIC, y el porcentaje de mujeres en las actividades TIC es mayor que el porcentaje de mujeres en las actividades no TIC. Además, en los dos tipos de actividades la evolución del colectivo femenino ha sido positiva, ya que han ganado peso específico con respecto a los hombres entre 2002 y 2007.

Vamos a estudiar cómo se concentran los empleados TIC y no TIC en las diferentes actividades. Al igual que hicimos en el anterior epígrafe, el análisis lo vamos a diferenciar entre el total de la población, el colectivo masculino y el colectivo femenino. En los siguientes cuadros 7 y 8 exponemos los datos.

CUADRo 6.- - Índices de participación masculina y femenina de los ocupados TIC y no TIC por sectores de actividad en el año 2002 y 2007

\begin{tabular}{|c|c|c|}
\hline & \multicolumn{2}{|c|}{ Ocupados TIC (por sectores de actividad) } \\
\hline & 2002 & 2007 \\
\hline HOMBRES & $57,68 \%$ & $54,70 \%$ \\
\hline \multirow[t]{3}{*}{ MUJERES } & $42,32 \%$ & $45,30 \%$ \\
\hline & \multicolumn{2}{|c|}{ Ocupados no TIC (por sectores de actividad) } \\
\hline & 2002 & 2007 \\
\hline HOMBRES & $62,67 \%$ & $59,28 \%$ \\
\hline MUJERES & $37,32 \%$ & $40,72 \%$ \\
\hline
\end{tabular}

CUADRO 7.-Índices de concentración para el total de la población, hombres y mujeres en el año 2002 y 2007 , para las actividades con ocupados TIC

\begin{tabular}{|c|c|c|c|c|c|}
\hline \multicolumn{2}{|c|}{ ESPAÑA } & \multicolumn{2}{c|}{ HOMBRES } & \multicolumn{2}{c|}{ MUJERES } \\
\hline Administración Pública & $8,38 \%$ & Intermediación monetaria & $6,95 \%$ & Administración Pública & $10,53 \%$ \\
\hline $\begin{array}{c}\text { Actividades jurídicas, } \\
\text { consulta, asesoramiento y } \\
\text { gestión empresarial }\end{array}$ & $7,63 \%$ & Administración Pública & $6,80 \%$ & $\begin{array}{c}\text { Actividades jurídicas, } \\
\text { consulta, asesoramiento y } \\
\text { gestión empresarial }\end{array}$ & $9,16 \%$ \\
\hline $\begin{array}{c}\text { Intermediación monetaria } \\
\text { Las 5 actividades con } \\
\text { mayor concetración }\end{array}$ & $6,02 \%$ & $\begin{array}{c}\text { Actividades jurídicas, } \\
\text { consulta, asesoramiento y } \\
\text { gestión empresarial }\end{array}$ & $6,50 \%$ & Intermediación monetaria & $4,74 \%$ \\
\hline
\end{tabular}


CuAdro 7 (cont.).- - Índices de concentración para el total de la población, hombres y mujeres en el año 2002 y 2007, para las actividades con ocupados TIC

\begin{tabular}{|c|c|c|c|c|c|}
\hline \multicolumn{6}{|c|}{2007} \\
\hline \multicolumn{2}{|l|}{ ESPAÑA } & \multicolumn{2}{|l|}{ HOMBRES } & \multicolumn{2}{|l|}{ MUJERES } \\
\hline Administración Pública & $8,12 \%$ & $\begin{array}{l}\text { Actividades jurídicas, } \\
\text { consulta, asesoramiento y } \\
\text { gestión empresarial }\end{array}$ & $6,19 \%$ & Administración Pública & $10,82 \%$ \\
\hline $\begin{array}{l}\text { Actividades jurídicas, } \\
\text { consulta, asesoramiento y } \\
\text { gestión empresarial }\end{array}$ & $7,95 \%$ & Intermediación monetaria & $5,93 \%$ & $\begin{array}{l}\text { Actividades jurídicas, } \\
\text { consulta, asesoramiento y } \\
\text { gestión empresarial }\end{array}$ & $10,07 \%$ \\
\hline Intermediación monetaria & $5,46 \%$ & Administración Pública & $5,89 \%$ & Intermediación monetaria & $4,90 \%$ \\
\hline $\begin{array}{l}\text { Las } 5 \text { actividades con } \\
\text { mayor concetración }\end{array}$ & $29,69 \%$ & $\begin{array}{l}\text { Las } 5 \text { actividades con } \\
\text { mayor concetración }\end{array}$ & $27,71 \%$ & $\begin{array}{l}\text { Las } 5 \text { actividades con } \\
\text { mayor concetración }\end{array}$ & $33,76 \%$ \\
\hline
\end{tabular}

En definitiva, podemos comprobar que existen tres actividades en las que se desarrolla una parte muy destacada del empleo considerado como TIC. Por otro lado, las mujeres de nuevo se concentran en mayor medida que los hombres en estas actividades.

Por tanto, cabe afirmar que el empleo no TIC masculino está enfocado generalmente a actividades relacionadas con el transporte y a la construcción, mientras que el empleo no TIC femenino se reparte mayoritariamente en empleo de hogar, sanitario y de comercio.

CuAdro 8.--índices de concentración para el total de la población, hombres y mujeres en el año 2002 y 2007, para las actividades con ocupados no TIC

\begin{tabular}{|c|c|c|c|c|c|}
\hline \multicolumn{2}{|c|}{ ESPAÑA } & \multicolumn{2}{c|}{ HOMBRES } & \multicolumn{2}{c|}{ MUJERES } \\
\hline $\begin{array}{c}\text { Construcción general } \\
\text { de inmuebles y obras de } \\
\text { ingeniería civil }\end{array}$ & $9,25 \%$ & $\begin{array}{c}\text { Construcción general } \\
\text { de inmuebles y obras de } \\
\text { ingeniería civil }\end{array}$ & $14,60 \%$ & $\begin{array}{c}\text { Hogares que emplean } \\
\text { personal doméstico }\end{array}$ & $9,54 \%$ \\
\hline $\begin{array}{c}\text { Otro comercio al por } \\
\text { menor de artículos nuevos } \\
\text { en establecimientos } \\
\text { especializados }\end{array}$ & $5,23 \%$ & $\begin{array}{c}\text { Otros tipos de transporte } \\
\text { terrestre }\end{array}$ & $5,72 \%$ & Actividades sanitarias & $8,83 \%$ \\
\hline $\begin{array}{c}\text { Actividades sanitarias } \\
\text { Las } 5 \text { actividades con } \\
\text { mayor concetración }\end{array}$ & $4,67 \%$ & $\begin{array}{c}\text { Acabado de edificios y } \\
\text { obras }\end{array}$ & $3,98 \%$ & $\begin{array}{c}\text { Otro comercio al por } \\
\text { menor de artículos nuevos } \\
\text { en establecimientos } \\
\text { especializados }\end{array}$ & $8,44 \%$ \\
\hline \multicolumn{2}{|c|}{$26,94 \%$} & $\begin{array}{c}\text { Las } 5 \text { actividades con } \\
\text { mayor concetración }\end{array}$ & $31,26 \%$ & $\begin{array}{c}\text { Las 5 actividades con } \\
\text { mayor concetración }\end{array}$ & $37,15 \%$ \\
\hline
\end{tabular}


Cuadro 8 (cont.).-Índices de concentración para el total de la población, hombres y mujeres en el año 2002 y 2007, para las actividades con ocupados no TIC

\begin{tabular}{|c|c|c|c|c|c|}
\hline \multicolumn{2}{|c|}{ ESPAÑA } & \multicolumn{2}{c|}{ HOMBRES } & \multicolumn{2}{c|}{ MUJERES } \\
\hline $\begin{array}{c}\text { Construcción general } \\
\text { de inmuebles y obras de } \\
\text { ingeniería civil }\end{array}$ & $10,37 \%$ & $\begin{array}{c}\text { Construcción general } \\
\text { de inmuebles y obras de } \\
\text { ingeniería civil }\end{array}$ & $17,25 \%$ & $\begin{array}{c}\text { Hogares que emplean } \\
\text { personal doméstico }\end{array}$ & $11,41 \%$ \\
\hline $\begin{array}{c}\text { Hogares que emplean } \\
\text { personal doméstico }\end{array}$ & $5,04 \%$ & $\begin{array}{c}\text { Otros tipos de transporte } \\
\text { terrestre }\end{array}$ & $5,34 \%$ & Actividades sanitarias & $8,65 \%$ \\
\hline $\begin{array}{c}\text { Otro comercio al por } \\
\text { menor de artículos nuevos } \\
\text { en establecimientos } \\
\text { especializados }\end{array}$ & $4,90 \%$ & $\begin{array}{c}\text { Instalaciones de edificios } \\
\text { y obras }\end{array}$ & $4,14 \%$ & $\begin{array}{c}\text { Otro comercio al por } \\
\text { menor de artículos nuevos } \\
\text { en establecimientos } \\
\text { especializados }\end{array}$ & $7,47 \%$ \\
\hline $\begin{array}{c}\text { Las } 5 \text { actividades con } \\
\text { mayor concetración }\end{array}$ & $29,86 \%$ & $\begin{array}{c}\text { Las } 5 \text { actividades con } \\
\text { mayor concetración }\end{array}$ & $34,41 \%$ & $\begin{array}{c}\text { Las 5 actividades con } \\
\text { mayor concetración }\end{array}$ & $39,14 \%$ \\
\hline
\end{tabular}

A partir del análisis anterior, analizaremos seguidamente el nivel de segregación que presentan las actividades en las que trabajan los ocupados españoles. Teniendo presentes los índices ya calculados anteriormente, será interesante comprobar y mediante los datos que se presentan a continuación si las actividades no relacionadas con las TIC presentan mayor nivel de segregación que las que se relacionan con las TIC.

CuAdro 9.-Evolucion de los índices ID e IP para los ocupados TIC y no TIC por sectores de actividad en el año 2002 y 2007

\begin{tabular}{|l|c|c|}
\cline { 2 - 3 } \multicolumn{1}{c|}{} & \multicolumn{2}{c|}{ Índice de Duncan y Duncan } \\
\cline { 2 - 3 } \multicolumn{1}{c|}{} & 2002 & 2007 \\
\hline $\begin{array}{l}\text { Ocupados TIC (por sectores } \\
\text { de actividad) }\end{array}$ & 0,2390 & 0,2454 \\
\hline $\begin{array}{l}\text { Ocupados no TIC (por } \\
\text { sectores de actividad) }\end{array}$ & 0,5508 & 0,5597 \\
\hline \multirow{2}{*}{$\begin{array}{l}\text { Ocupados TIC (por sectores } \\
\text { de actividad) }\end{array}$} & \multicolumn{2}{|c|}{ Índice de Karmel y Maclachlan } \\
\hline \multirow{2}{*}{$\begin{array}{l}\text { Ocupados no TIC (por } \\
\text { sectores de actividad) }\end{array}$} & 0,2002 & 2007 \\
\hline
\end{tabular}


El cuadro 9 recoge los resultados obtenidos, que conducen a las siguientes conclusiones:

- Para los ocupados TIC, según el ID en el año 2002, un 23,9 por ciento de hombres (o de mujeres) deberían cambiar de actividad para que ambos sexos estuvieran igual distribuidos por actividades. En el año 2007 este porcentaje aumentó a 24,54 por ciento. Según el índice IP, en el año 2002, un 20,96 por ciento de los puestos de trabajo deberían pasar de mujeres a hombres ( $\mathrm{y}$ viceversa) para que el grado de segregación fuera nulo; en el año 2007, este porcentaje había aumentado a 24,03 por ciento.

- Para los ocupados no TIC, según el ID en el año 2002 un 55,08 por ciento de hombres (o de mujeres) deberían cambiar de actividad para que ambos sexos estuvieran igual distribuidos por actividades. En el año 2007 este porcentaje aumentó a 55,97 por ciento. Según el índice IP, en el año 2002, un 36,84 por ciento de los puestos de trabajo deberían pasar de mujeres a hombres (y viceversa) para que el grado de segregación fuera nulo; en el año 2007, este porcentaje había aumentado a 40,75 por ciento.

- La segregación aumenta tanto en los sectores de actividad con ocupados TIC como en los relacionados con ocupados no TIC.

- Si bien el Índice de Duncan y Duncan no permite la comparación entre variables con distinto número de actividades, es decir, comparaciones entre TIC y no TIC, el Índice de Karmel y MacLachlan sí nos permite concluir que la segregación por actividades es mucho mayor para el empleo no TIC que para el empleo TIC, tanto en el año 2002 como en el 2007, e igualmente poder subrayar un mayor crecimiento de la segregación en las actividades no relacionadas con los ocupados TIC que en las actividades con ocupados TIC.

Para finalizar el análisis horizontal del empleo TIC, pasamos a continuación a ver cómo se distribuye dicho empleo en las distintas actividades económicas. Con este objetivo estableceremos qué actividades están masculinizadas o feminizadas, considerando al resto como actividades integradas. Para ello, seguimos manteniendo el mismo ratio que ya dispusimos cuando analizamos la distribución para las ocupaciones TIC ${ }^{5}$.

En el año 2002, la distribución sectorial del empleo TIC implicaba que 55 las actividades con ocupados TIC que estaban masculinizadas, es decir, un 27,09 por ciento,

${ }^{5}$ Se han omitido los cuadros por razones de espacio. 
mientras que en el 2007 habían aumentado a 64, que representan un 30,77 por ciento del total. Por el contrario, en el año 2002 eran 48 las actividades con ocupados TIC que estaban feminizadas, es decir, un 23,65 por ciento, incrementándose a 49 en el 2007, que suponen el 23,56 por ciento del total. Por tanto, en el año 2002 eran 100 las actividades con ocupados TIC que estaban integradas, es decir, un 49,26 por ciento. En el año 2007 las actividades integradas descendían a 95 por lo que el porcentaje disminuyó a 45,67 por ciento.

Cuando analizamos la distribución sectorial del empleo no TIC, en el año 2002 eran 107 las que se encontraban masculinizadas, lo cual supone un 52,71 por ciento del total, en tanto que en el año 2007 se había producido un ligero empeoramiento en términos absolutos en cuanto a la distribución ya que eran 109 actividades, es decir, un 52,40 por ciento. Por la parte de las mujeres, tanto en el año 2002 como en el 2007 únicamente había 41 actividades feminizadas, es decir, el 20,2 por ciento y el 19,71 por ciento, respectivamente. Estos datos nos dejan 55 actividades con ocupados no TIC integradas en el 2002, que suponen el 27,09 por ciento, aumentando a 58 en 2007, un 27,88 por ciento.

En el gráfico 2 hemos recogido los resultados y la evolución de la distribución de las actividades TIC y no TIC entre 2002 y 2007 . Se hace evidente una mejor distribución por género en ambos años del mercado de trabajo en las actividades que tienen ocupados TIC, ya que en éstas el mayor porcentaje corresponde a las actividades integradas, mientras que en las no TIC son más importantes las actividades que están masculinizadas.

GRÁfICo 2.-Evolución en porcentaje de los sectores de actividad masculinizados, feminizados e integrados, con ocupados TIC y no TIC, en 2002 y 2007. (Fuente: elaboración propia a partir de la EPA)

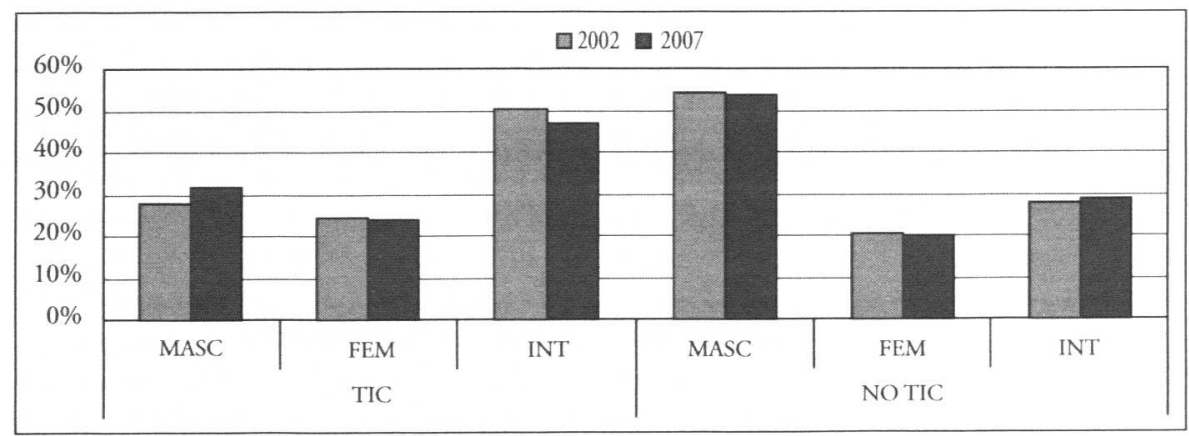


En conclusión, de los análisis efectuados hasta el momento queremos destacar los siguientes resultados:

- Las mujeres presentan en España una mayor relación que los hombres con el empleo TIC. Con datos del 2007, mientras que alrededor del 23 por ciento de las mujeres que trabajaban lo hacían en este tipo de ocupaciones laborales, los hombres limitaban este porcentaje al 20 por ciento.

- Además, cuando medimos la segregación desde una perspectiva vertical (ocupaciones laborales), se concluye como el empleo relacionado con las TIC reduce notablemente esta desigualdad laboral por género (casi un 25 por ciento menos).

- Sin embargo, esta situación continua siendo elevada también para las ocupaciones TIC. Según el ID calculado, en el año 2002 un 44,78 por ciento de hombres (o de mujeres) deberían cambiar de ocupación para que ambos sexos estuvieran igual distribuidos por ocupaciones. En el año 2007 este porcentaje incluso aumentó hasta el 45,93 por ciento.

- Cuando se adopta una perspectiva horizontal (sectores de actividad) para analizar las diferencias laborales por género, los resultados anteriores se ven corroborados. La segregación laboral por género es mayor entre las ramas de actividad para el empleo no TIC, de forma que estas tecnologías reducen la magnitud de esta situación. La evolución de este indicador es más favorable entre las actividades TIC. Pero, a pesar de ello, los niveles de segregación continúan siendo elevados, también para las mujeres que desempeñan tareas relacionadas con las TIC en las actividades más relacionadas con este tipo de tecnologías ( 0,23 en 2002 y 0,24 en 2007).

- Las nuevas tecnologías no consiguen suprimir, por tanto, las desventajas laborales que deben afrontar las mujeres. A pesar de ello, debe destacarse el hecho de que este tipo de empleo reduce en cierta medida la magnitud de este tipo de diferencia laboral por género, por lo que la mujer, aunque no disfruta de situaciones de igualdad laboral, si que se enfrenta a un contexto notablemente más favorable.

\section{LOS DETERMINANTES DEL EMPLEO TIC}

Como se ha puesto de manifiesto hasta el momento, la posición laboral femenina presenta diferencias con respecto a la posición laboral masculina en el empleo rela- 
cionado con las TIC, o dicho de otra forma, hombres y mujeres no se relacionan de la misma forma con las ocupaciones TIC. En este apartado pretendemos profundizar en estas desigualdades, identificando las variables laborales y personales que determinan la empleabilidad en el empleo TIC y mostrar cuáles son las diferencias existentes por género.

Para ello, se desarrolla una serie de modelos logit sobre la probabilidad de estar empleado en una actividad u ocupación considerada como TIC frente a estar empleado en otro tipo de actividad u ocupación ${ }^{6}$. Las estimaciones se especifican para la población total, para los hombres y para las mujeres, con el objetivo de intentar detectar, en la medida de lo posible, las diferencias existentes por género.

El modelo desarrollado se basa en las siguientes ecuaciones:

$$
Y_{i}=\frac{1}{1+e^{-\alpha-\beta_{j} X_{i j}}}+u_{i} \quad ; \quad \operatorname{Prob}\left(Y_{i}\right)=\frac{1}{1+e^{-\left(\alpha-\beta_{j} X_{i j}\right)}}+u_{i}
$$

donde $Y_{i}$ representa el suceso a contrastar, en nuestro caso, estar empleado en una actividad u ocupación considerada como TIC frente a estar empleado en el resto de actividades u ocupaciones, y $\operatorname{Prob}\left(Y_{i}=1\right)$ es la probabilidad de que se produzca dicho suceso. Las variables $X_{i j}$ recogen las características personales y laborales determinantes del suceso a contrastar. Para este trabajo se han considerado las siguientes:

- Variables personales como la edad; el estado civil; el nivel de formación; la comunidad autónoma de residencia (CCAA, en adelante) ${ }^{7}$; y ser madre o padre según el género del individuo considerado.

${ }^{6}$ Se utilizan las dos definiciones apuntadas en la introducción del artículo.

7 Para construir la variable CCAA, las 17 categorías se han agregado en 3 en función de la intensidad con que presenten actividades u ocupaciones TIC, obteniendo la siguiente clasificación para sectores de actividad: Murcia, Extremadura, Canarias, Andalucía, Valencia y Navarra con escasa presencia de actividades TIC; Galicia, Baleares, Castilla la Mancha, Asturias y Castilla León con presencia media de actividades TIC; La Rioja, Cantabria, Cataluña, Aragón, País Vasco y Madrid con elevada presencia de actividades TIC. Y para ocupaciones: Extremadura, Castilla la Mancha, Andalucía, Canarias, La Rioja y Murcia con escasa presencia de ocupaciones TIC; Castilla León, Galicia, Asturias, Cantabria y Valencia con presencia media de ocupaciones TIC; Baleares, Navarra, Aragón, País Vasco, Cataluña y Madrid con elevada presencia de ocupaciones TIC. 
- Variables profesionales como la duración de la jornada laboral (completa o parcial); una variable que resume la situación laboral que se mantiene en el mercado de trabajo junto con la duración del contrato para aquellos que sean asalariados; la ocupación cuando se analice la posibilidad de estar empleado en una actividad TIC; y el sector de actividad cuando se analice la posibilidad de estar empleado en una ocupación TIC.

Los datos utilizados en las estimaciones provienen de la EPA del año 2007, último año disponible en el momento de la elaboración de este trabajo.

\subsection{Variables determinantes del empleo TIC desde la perspectiva de los sectores de actividad ${ }^{8}$}

El primero de los modelos estimados utiliza criterios de sector de actividad para la definición del empleo TIC, y estima la probabilidad de trabajar en una actividad TIC frente a hacerlo en el resto de la estructura productiva. Sus resultados no son excesivamente consistentes (cuadro 10). Los estadísticos sobre la bondad del ajuste (incluidos al final del cuadro) indican que las estimaciones no son demasiado robustas, sobre todo, en el caso de las mujeres. Este hecho puede estar motivado por la escasa presencia del suceso a contrastar dentro de dicho colectivo, es decir, el número de mujeres trabajando en sectores TIC. No obstante, cabe subrayar los siguientes resultados:

- Ser joven (entre 16 y 35 años) incrementa la probabilidad de trabajar en sectores TIC, tanto para el empleo total como en la diferenciación por género, si bien esta probabilidad es mayor en el caso de los hombres que en el caso de las mujeres.

- El estado civil es una variable que no resulta significativa, ni para el total del empleo, ni para hombres ni para mujeres.

— La formación es una de las características personales más determinante. Una mayor educación incrementa la probabilidad de trabajar en sectores TIC, más en el caso de las mujeres que en el caso de los hombres. 
- Es más probable estar empleado en el empleo TIC en aquellas CCAA que cuentan con una elevada presencia de sectores TIC, probabilidad más favorable en el caso de los ocupados que en el caso de las ocupadas.

- La variable «convivir con hijos en el hogar» no resulta significativa.

- La duración de la jornada laboral resulta muy determinante respecto de la asignación a las ramas TIC, y se concreta en la exigencia del desarrollo de jornadas a tiempo completo. Esta variable sólo es significativa en el caso del empleo total, ya que ni para hombres ni para mujeres es una variable trascendente.

- Ser asalariado indefinido es la situación que más incrementa la probabilidad de trabajar en sectores económicos TIC para los tres tipos de población estudiada.

- La experiencia profesional incrementa la probabilidad de estar empleado en un sector TIC, de modo que haber estado empleado por más de 58 meses $^{9}$ en la empresa actual determina la mayor probabilidad de estar contratado en sectores TIC, siendo más probable para las mujeres que para los hombres.

- Frente al resto de posibilidades, desempeñar ocupaciones laborales no manuales cualificadas ${ }^{10}$ estimula la probabilidad de trabajar en el empleo TIC.

- Es interesante la probabilidad asociada a la constante ya que en todos los casos su signo es negativo, lo cual significa que, al margen de las variables estudiadas, es más probable trabajar en sectores de actividad que no tienen relación con el empleo TIC. Esta probabilidad es mayor en el caso de las mujeres que en el caso de los hombres.

CUADRo 10.- Modelo sobre la probabilidad de estar empleado en una actividad considerada como TIC frente a estar empleado en el resto de actividades. (Fuente: elaboración propia a partir de la EPA)

\begin{tabular}{|l|c|c|c|c|c|c|}
\hline & \multicolumn{2}{|c|}{ TOTAL } & \multicolumn{2}{c|}{ MUJERES } & \multicolumn{2}{c|}{ HOMBRES } \\
\hline & B & Sig. & B & Sig. & B & Sig. \\
\hline Edad por tramos & & 0 & & $0,049^{* *}$ & & 0 \\
\hline
\end{tabular}

9 Se ha escogido 58 meses por ser la media de la distribución considerada, en este caso, la duración en meses de la experiencia laboral en su empresa actual.

${ }^{10}$ La ocupación laboral ha sido categorizada de la siguiente manera: ocupaciones no manuales de alta cualificación (White Collar High Skill-WCHS), ocupaciones no manuales de baja cualificación (White Collar Low Skill-WCLS), ocupaciones manuales de alta cualificación (Blue Collar High Skill-BCHS) y ocupaciones manuales de baja cualificación (Blue Collar Low Skill-BCLS). 
Cuadro 10 (cont.).- - Modelo sobre la probabilidad de estar empleado en una actividad considerada como TIC frente a estar empleado en el resto de actividades. (Fuente: elaboración propia a partir de la EPA)

\begin{tabular}{|c|c|c|c|c|c|c|}
\hline & \multicolumn{2}{|c|}{ TOTAL } & \multicolumn{2}{|c|}{ MUJERES } & \multicolumn{2}{|c|}{ HOMBRES } \\
\hline & $\mathrm{B}$ & Sig. & B & Sig. & B & Sig. \\
\hline Jóvenes (16-35 años) & 0,415 & 0 & 0,413 & 0,037 & 0,506 & 0 \\
\hline Maduros/as (más de 55) & $-0,335$ & $0,085^{* *}$ & $-0,415$ & $0,344^{* *}$ & $-0,374$ & $0,087^{* *}$ \\
\hline Estado civil & & $0,223^{* *}$ & & $0,756^{* *}$ & & $0,429 * *$ \\
\hline Soltero/a & 0,054 & $0,617^{* *}$ & 0,009 & $0,961^{* *}$ & 0,112 & $0,400^{* *}$ \\
\hline Otros casos & $-0,378$ & $0,114^{* *}$ & $-0,264$ & $0,466^{* *}$ & $-0,285$ & $0,376^{* *}$ \\
\hline Formación & & 0 & & 0,013 & & 0,001 \\
\hline Ed. Primaria & $-0,845$ & 0,001 & $-1,231$ & $0,053^{* *}$ & $-0,72$ & 0,013 \\
\hline Ed. Superior & 0,274 & 0,009 & 0,39 & 0,043 & 0,317 & 0,01 \\
\hline Tipo de CCAA & & 0 & & 0 & & 0 \\
\hline Escasa presencia de act. TIC & $-0,292$ & 0,042 & $-0,523$ & $0,051^{* *}$ & $-0,223$ & $0,194^{* *}$ \\
\hline Elevada presencia de act. TIC & 0,579 & 0 & 0,524 & 0,015 & 0,601 & 0 \\
\hline Padres/Madres (SI) & 0,199 & $0,098^{* *}$ & 0,049 & $0,809^{* *}$ & 0,038 & $0,810^{* *}$ \\
\hline $\begin{array}{l}\text { Duración Jornada laboral } \\
\text { (parcial) }\end{array}$ & $-0,573$ & 0,001 & $-0,442$ & $0,065^{* *}$ & $-0,422$ & $0,142 * *$ \\
\hline Situación profesional & & 0 & & 0,009 & & 0 \\
\hline $\begin{array}{l}\text { Asalariado con un contrato } \\
\text { temporal }\end{array}$ & $-0,602$ & 0 & $-0,524$ & 0,011 & $-0,585$ & 0 \\
\hline No asalariado & $-0,719$ & 0 & $-0,64$ & 0,036 & $-0,847$ & 0 \\
\hline $\begin{array}{l}\text { Experiencia profesional en la } \\
\text { empresa (+ } 58 \text { meses) }\end{array}$ & 0,331 & 0,001 & 0,38 & 0,048 & 0,306 & 0,015 \\
\hline Ocupación laboral agregada & & 0 & & 0,015 & & 0 \\
\hline WCLS & $-1,09$ & 0 & $-0,423$ & 0,03 & $-1,4$ & 0 \\
\hline BCHS & $-1,054$ & 0 & $-0,387$ & $0,489 * *$ & $-1,425$ & 0 \\
\hline BCLS & $-1,591$ & 0 & $-0,998$ & 0,002 & $-1,919$ & 0 \\
\hline Constante & $-3,397$ & 0 & $-4,003$ & 0 & $-2,993$ & 0 \\
\hline CASOS & \multicolumn{2}{|c|}{69.575} & \multicolumn{2}{|c|}{29.233} & \multicolumn{2}{|c|}{40.342} \\
\hline$-2 \log$ de la verosimilitud & \multicolumn{2}{|c|}{$4.685,91$} & \multicolumn{2}{|c|}{$1.526,75$} & \multicolumn{2}{|c|}{$3.072,30$} \\
\hline R cuadrado de Cox y Snell & \multicolumn{2}{|c|}{0,028} & \multicolumn{2}{|c|}{0,016} & \multicolumn{2}{|c|}{0,041} \\
\hline R cuadrado de Nagelkerke & \multicolumn{2}{|c|}{0,123} & \multicolumn{2}{|c|}{0,087} & \multicolumn{2}{|c|}{0,157} \\
\hline Porcentaje de clasificación & \multicolumn{2}{|c|}{97,1} & \multicolumn{2}{|c|}{98,0} & \multicolumn{2}{|c|}{96,5} \\
\hline
\end{tabular}


CUadro 10 (cont.).--Modelo sobre la probabilidad de estar empleado en una actividad considerada como TIC frente a estar empleado en el resto de actividades. (Fuente: elaboración propia a partir de la EPA)

\begin{tabular}{|l|c|c|c|c|c|c|}
\hline & \multicolumn{2}{|c|}{ TOTAL } & \multicolumn{2}{c|}{ MUJERES } & \multicolumn{2}{c|}{ HOMBRES } \\
\hline & B & Sig. & B & Sig. & B & Sig. \\
\hline $\begin{array}{l}\text { Sig. Hosmer-Lemeshow } \\
\text { (8 gl.) }\end{array}$ & \multicolumn{2}{|c|}{0.815} & \multicolumn{2}{|c|}{0.963} & \multicolumn{2}{|c|}{0,989} \\
\hline
\end{tabular}

* Persona de referencia: de edad intermedia (36-54 años), casado/a, con educación secundaria, que vive en una CCAA con una presencia media de actividades TIC, se encuentra ocupado como asalariado con un contrato indefinido desarrollando una ocupación no manual de elevada cualificación (White collar high skill-WCHS).

** Valores no significativos bajo una probabilidad del $95 \%$.

Como resumen, podemos decir que el desarrollo de las actividades TIC parece exigir, por tanto, individuos jóvenes, con altos niveles de estudios, ubicados en CCAA que ya tengan un alta presencia de actividades TIC, una dedicación completa y estable en el tiempo, lo que se asocia con elevados niveles de experiencia profesional, y el desarrollo de tareas no manuales cualificadas.

\subsection{Variables determinantes del empleo TIC desde la perspectiva de los sectores de las ocupaciones laborales}

Complementariamente a la perspectiva utilizada en el apartado anterior, el empleo TIC se puede identificar también como aquel que supone el desempeño de ciertas ocupaciones laborales relacionadas con las TIC. Considerando esta perspectiva, ahora vamos a estimar los modelos anteriores pero definiendo la variable dependiente (trabajar en el empleo TIC) de acuerdo con el criterio de Empleo TIC - ocupaciones. A partir de los resultados obtenidos, presentados en el cuadro número 11, queremos destacar la obtención de mejores estadísticos de ajuste que los obtenidos en las actividades TIC, debido, en parte, a que en este caso el tamaño de la población estudiada es sensiblemente mayor.

En relación con el total de la población, como parámetros generales que conducen al desarrollo de un empleo con ocupaciones TIC, se pueden extraer los siguientes resultados:

- La edad no arroja conclusiones definitivas para determinar que sea una variable importante a la hora de trabajar en ocupaciones TIC. 
- De nuevo, el estado civil no resulta significativo, ni para el total de la población, ni para hombres ni para mujeres.

- La educación superior es una de las características más determinantes para que una persona, ya sea hombre o mujer, se encuentre empleada en ocupaciones TIC, de manera que poseer estudios superiores se constituye en el principal requerimiento para el desarrollo de este tipo de ocupaciones, si bien es más determinante en el caso de los hombres que de las mujeres.

- Por supuesto, vivir en una CCAA donde existe una mayor presencia de ocupaciones TIC facilita por si solo que un individuo se emplee dentro de ocupaciones TIC.

- La maternidad y la paternidad, tal como ha sido definida, es decir, considerando aquellas personas que conviven en un hogar con hijos, no resulta significativa.

CUADro 11.-Modelo sobre la probabilidad de estar empleado en una ocupación considerada como TIC frente a estar empleado en el resto de ocupaciones. (Fuente: elaboración propia a partir de la EPA)

\begin{tabular}{|l|c|c|c|c|c|c|}
\hline & \multicolumn{2}{|c|}{ TOTAL } & \multicolumn{2}{c|}{ MUJERES } & \multicolumn{2}{c|}{ HOMBRES } \\
\hline & B & Sig. & B & Sig. & B & Sig. \\
\hline Edad por tramos & & 0,045 & & $0,084^{* *}$ & & 0,003 \\
\hline Jóvenes (16-35 años) & 0,055 & $0,241^{* *}$ & 0,144 & 0,037 & $-0,037$ & $0,576^{* *}$ \\
\hline Maduros/as (más de 55) & 0,157 & 0,018 & $-0,047$ & $0,686^{* *}$ & 0,271 & 0,001 \\
\hline Estado civil & & $0,132^{* *}$ & & $0,063^{* *}$ & & $0,640^{* *}$ \\
\hline Soltero/a & $-0,06$ & $0,203^{* *}$ & $-0,068$ & $0,341^{* *}$ & $-0,062$ & $0,351^{* *}$ \\
\hline Otros casos & $-0,139$ & $0,086^{* *}$ & $-0,248$ & 0,023 & $-0,017$ & $0,892^{* *}$ \\
\hline Formación & & 0 & & 0 & & 0 \\
\hline Ed. Primaria & $-1,502$ & 0 & $-1,739$ & 0 & $-1,32$ & 0 \\
\hline Ed. Superior & 1,112 & 0 & 0,827 & 0 & 1,287 & 0 \\
\hline Tipo de CCAA & & 0 & & 0 & & 0 \\
\hline Escasa presencia de ocup. TIC & 0,006 & $0,914^{* *}$ & 0,086 & $0,282^{* *}$ & $-0,038$ & $0,600^{* *}$ \\
\hline Elevada presencia de ocup. TIC & 0,345 & 0 & 0,364 & 0 & 0,339 & 0 \\
\hline Padres/Madres (SI) & $-0,025$ & $0,587^{* *}$ & 0,046 & $0,493^{* *}$ & $-0,035$ & $0,606^{* *}$ \\
\hline Duración Jornada laboral (parcial) & $-0,353$ & 0 & $-0,432$ & 0 & $-0,426$ & 0,002 \\
\hline Situación profesional & & 0 & & 0 & & 0 \\
\hline Asalariado con un contrato temporal & $-0,719$ & 0 & $-0,706$ & 0 & $-0,7$ & 0 \\
\hline
\end{tabular}


CUADRo 11 (cont.).--Modelo sobre la probabilidad de estar empleado en una ocupación considerada como TIC frente a estar empleado en el resto de ocupaciones. (Fuente: elaboración propia a partir de la EPA)

\begin{tabular}{|c|c|c|c|c|c|c|}
\hline & \multicolumn{2}{|c|}{ TOTAL } & \multicolumn{2}{|c|}{ MUJERES } & \multicolumn{2}{|c|}{ HOMBRES } \\
\hline & B & Sig. & B & Sig. & B & Sig. \\
\hline No asalariado & $-0,134$ & 0,009 & $-0,286$ & 0,001 & $-0,078$ & $0,232 * *$ \\
\hline $\begin{array}{l}\text { Experiencia profesional en la } \\
\text { empresa (+ } 58 \text { meses) }\end{array}$ & $-0,062$ & $0,164 * *$ & $-0,196$ & 0,003 & 0,038 & $0,539 * *$ \\
\hline Actividad laboral agregada & & 0 & & 0 & & 0 \\
\hline Industria & 1,617 & 0 & 1,541 & 0 & 1,714 & 0 \\
\hline Construcción & 1,119 & 0 & 3,48 & 0 & 0,984 & 0 \\
\hline Servicios & 1,554 & 0 & 0,852 & 0,004 & 1,959 & 0 \\
\hline Constante & $-3,054$ & 0 & $-2,279$ & 0 & $-3,468$ & 0 \\
\hline CASOS & \multicolumn{2}{|c|}{69.884} & \multicolumn{2}{|c|}{29.268} & \multicolumn{2}{|c|}{40.616} \\
\hline$-2 \log$ de la verosimilitud & \multicolumn{2}{|c|}{$18.318,55$} & \multicolumn{2}{|c|}{$7.926,90$} & \multicolumn{2}{|c|}{$9.934,42$} \\
\hline R cuadrado de Cox y Snell & \multicolumn{2}{|c|}{0,123} & \multicolumn{2}{|c|}{0,128} & \multicolumn{2}{|c|}{0,149} \\
\hline R cuadrado de Nagelkerke & \multicolumn{2}{|c|}{0,19} & \multicolumn{2}{|c|}{0,192} & \multicolumn{2}{|c|}{0,237} \\
\hline Porcentaje de clasificación & \multicolumn{2}{|c|}{78,6} & \multicolumn{2}{|c|}{78,1} & \multicolumn{2}{|c|}{80,0} \\
\hline Sig. Hosmer-Lemeshow (8 gl.) & \multicolumn{2}{|c|}{0.659} & \multicolumn{2}{|c|}{0.767} & \multicolumn{2}{|c|}{0.819} \\
\hline
\end{tabular}

* Persona de referencia: de edad intermedia (36-54 años), casado/a, con educación secundaria, que vive en una CCAA con una presencia media de actividades TIC, se encuentra ocupado como asalariado con un contrato indefinido desarrollando una ocupación no manual de elevada cualificación (White collar high skill-WCHS).

** Valores no significativos bajo una probabilidad del $95 \%$.

- El desarrollo de una jornada laboral a tiempo completo aumenta la probabilidad de que tanto hombres como mujeres desarrollen tareas de carácter TIC.

- Ser asalariado indefinido frente a otras situaciones laborales se asocia en mayor medida con las ocupaciones TIC.

- A diferencia del modelo anterior, ahora la experiencia en la empresa no resulta determinante para ubicarse en ocupaciones TIC.

- El desarrollo de las ocupaciones TIC se realiza en puestos de trabajo anexos a los sectores industriales (para el total de la población), a la construcción (para las mujeres) y terciarios (para los hombres). Ocupaciones y sectores laborales se encuentran íntimamente unidos y, en el caso de las TIC, se comprueba que dicha unión resulta muy intensa. 
- La constante presenta signo negativo, por lo que las conclusiones en este caso son idénticas a las obtenidas en el estudio por sectores de actividad.

En defnitiva, podemos decir que el desarrollo de las ocupaciones TIC parece exigir individuos con altos niveles de estudios, ubicados en CCAA que ya tengan un alta presencia de ocupaciones TIC, una dedicación completa a su empleo, ya sea en el sector industrial para el total de la población, en la construcción en el caso de las mujeres y en el sector servicios en el caso de los hombres.

\section{Conclusiones finales}

Después de analizar detalladamente la posición de hombres y mujeres, y teniendo en cuenta las hipótesis planteadas, no podemos concluir que el empleo TIC sea, definitivamente, un sector del mercado laboral en el que no se produzcan desigualdades por razón de género, si bien existen claros síntomas que hacen de este tipo de empleo un referente en el proceso social y económico que equipara mujeres y hombres en el mercado laboral.

Al margen de esta conclusión principal, podemos destacar los siguientes aspectos que resumen lo que aquí ha sido estudiado:

- El empleo TIC se encuentra aún en nuestro mercado laboral en una fase introductoria, ya que el porcentaje que ocupa respecto al empleo total no alcanza valores determinantes, por lo que el empleo no TIC sigue constituyendo el núcleo duro de nuestro mercado de trabajo. Al igual que sucede para el conjunto laboral, los hombres son mayoría en ambos tipos de empleo. Sin embargo, podemos encontrar diferencias significativas si analizamos con detenimiento el género, ya que el empleo TIC tiene mayor arraigo dentro de las mujeres que de los hombres. En este sentido, la población femenina progresa con paso más ligero que la población masculina, por lo que, si las mujeres siguen teniendo mayor peso que los hombres en los centros de cualificación, la madurez del empleo TIC puede producir un proceso de convergencia entre empleos masculinos y femeninos.

- No podemos concluir que la segregación dentro del empleo TIC favorezca la igualdad de género, si bien es muy importante el hecho de haber encontrado una parte de nuestro mercado de trabajo donde la segregación laboral por cuestión de género presenta valores significativamente menores a los del em- 
pleo no TIC. Este resultado se ve tremendamente reforzado al poder concluir que algunas ocupaciones que se encontraban tradicionalmente masculinizadas están convirtiéndose en integradas y no en ocupaciones feminizadas, lo cual sería indicativo de un proceso de re - segregación (Reskin y Roos, 1990), es decir, una dinámica en la que las mujeres están adoptando el papel de los hombres, y viceversa, lo cual no sería favorable a la lucha por la igualdad de género dentro de nuestro mercado laboral.

- A través de los modelos logit se ha comprobado como hombres y mujeres presentan características personales y laborales que determinan de forma desigual la probabilidad de estar empleado en los sectores u ocupaciones TIC. Este hecho, por si sólo, demuestra la desigualdad por género que existe en el mercado laboral español y, de forma concreta, si bien con un carácter menor, dentro del empleo TIC.

- Ha quedado perfectamente determinado el perfil de ocupado u ocupada dentro del empleo TIC. De esta forma, ser joven, tener una cualificación de grado superior, trabajar en una CCAA donde predomine el empleo TIC y tener contrato indefinido son las características que resumen el curriculum del empleado o empleada TIC. En este punto, queremos hacer hincapié en dos de ellas:

- La mayor probabilidad de desarrollar un trabajo TIC en las CCAA que ya tienen un alto nivel de este tipo de empleo hace que las TICs se focalicen en determinadas CCAA, atrofiando la capilaridad que requiere el desarrollo de la tecnología y de las comunicaciones para que se produzca un crecimiento real a nivel nacional de este tipo de empleo. En este sentido, las Administraciones Públicas de las CCAA deben desempeñar un papel fundamental en este proceso ya que en aquellas comunidades en las que no se ha experimentado el desarrollo del empleo TIC se debe redoblar el esfuerzo para fomentar e implementar este tipo de empleo. A partir de la clasificación que aquí se ha establecido, son Andalucía, Extremadura, Canarias y Murcia las que más deben esforzarse, teniendo como referencia el perfil que muestran otras regiones como Aragón, País Vasco, Cataluña y Madrid.

- La alta cualificación está definitivamente ligada al empleo TIC. En este orden, podemos considerar que variables como el número de alumnos y alumnas matriculados en las universidades españolas o el número de licenciados y licenciadas en los grados universitarios, entre otras, se configuran como verdaderas variables proxi que determinan el futuro de la igualdad 
de género dentro del empleo TIC. Por otro lado, el fomento de la oferta de empleo TIC que puedan llevar a cabo los responsables políticos no tiene sentido si, paralelamente, no se lleva a cabo el desarrollo, apoyo y promoción de los centros educativos, a cualquier nivel, que se encarguen de formar y cualificar a los demandantes de este tipo de empleo.

\section{REFERENCIAS BIBLIOGRÁFICAS}

Boisso, D.; Hayes, K.; Hirschberg, J. y Silber, J. (1994), «Occupational segregation in the multidimensional case: descomposition and tests of significance», Journal of Econometrics, núm. 61, págs. 161-171.

CÁCERES, J. I.; EsCOT, L. y SAIZ, J. (2004), «La segregación ocupacional y sectorial de la mujer en el mercado de trabajo español», Documento de trabajo, Facultad CCEE, Universidad Complutense de Madrid, 2004-2006.

CASTAÑo, C. (dir.) (2003), Indicadores laborales básicos de la situación de la mujer en España y sus regiones, Instituto de la Mujer y Ministerio de Igualdad.

Castaño, C.; Caprile, M. e Iglesias, C. (2005), «New work: old barriers but new opportunities for women», Documento de trabajo 05 / 200, Instituto de Análisis Económico y Social (IAES).

CAstells, M (2000), La era de la información, vol. I, La sociedad red, Alianza, Madrid.

CEBrián, I. y Moreno, G. (2008), «La situación de las mujeres en el mercado de trabajo español: desajustes y retos», Revista de Economía Industrial, núm. 367, págs. 121-137.

DE LA RicA, S. (2007), «Segregación ocupacional y diferencias salariales por género en España: 1995-2002», Documento de trabajo 2007-35, Serie Educación y Crecimiento, Cátedra FEDEA-Banco de España.

Ducatel, K. (ed.) (1994), Employment and technical change in Europe. Work organization, skills and training, Edward Elgar.

Duncan, O, y Duncan, J. (1955), «A methodological analysis of segregation indexes», American Sociological Review, núm. 20, págs. 210-217.

FLÜCKInger, Y. y SILber, J. (1999), The measurement of segregation in the labour force, Physica-Verlag Heidelberg, Nueva York.

Freeman, Ch.; Clarck, J. y Soete, L. (1985), Desempleo e innovación tecnológica, Ministerio de Trabajo y Seguridad Social.

Freeman, Ch. y Soete, L. (1996), Cambio tecnológico y empleo. Una estrategia de empleo para el siglo XXI, BT Telecomunicaciones y Fundación Universidad-Empresa. Madrid. 
IGLESIAS, C. (2005), La participación de la mujer en el mercado de trabajo nacional y madrileño, Instituto de Estudios Económicos, ISBN 84-88533-81-0.

Iglesias, C. y LloREnTE, R. (2009), «Evolución reciente de la segregación laboral por género en España», Documento de Trabajo del Instituto de Análisis Económico y Social (IAES).

KAKWANI, N. C. (1994), «Segregation by sex: measurement and hypothesis testing», Research on Economic Inequality, vol. 5.

Karmel, T. y Maclachlan, M. (1988), «Occupational sex segregation, increasing or decreasing?», The Economic Record, núm. 64, págs. 187-195.

Maté, J. L.; NAva, L. A. y Rodríguez, J. C. (2002), «La segregación ocupacional por razón de sexo en la economía española, 1994-1999», Revista del Ministerio de Trabajo y Asuntos Sociales, núm. 36, págs. 79-94.

OCDE (2004), OECD Information Technology Outlook 2004, París.

Otero, M. S. y Gradín, C. (2001), «Segregación ocupacional en España: una perspectiva territorial», Hacienda Pública Española, núm. 159-4, págs. 163-190.

RESKIN, B. F. y Roos, P. A. (1990), Job queues, gender queues: explining women's inroads into male occupations, Temple University Press, Philadelphia.

Salas, C. (2004), «Segregación y discriminación laboral por sexo», Documento de trabajo. Departamento de sociología, UAM, Iztapalapa, México.

SÁnchEZ, M. (1993), «La segregación de las mujeres en el mercado de trabajo español: 19641988», Hacienda Pública Española, núm. 124, págs. 123-139.

WATTs, M. J. (1992), «How should occupational gender segregation be measured?», Work, Employment and Society, núm. 6, págs. 474-487. 


\section{ANEXO}

A.1. Clasificación de empleo TIC por ocupaciones. (Fuente: $\mathrm{CNO}$ - 94)

\begin{tabular}{|c|c|}
\hline 111 & Dirección general y presidencia ejecutiva \\
\hline 112 & Dirección de departamento de producción \\
\hline 113 & Dirección de áreas y departamentos especializados \\
\hline 201 & \multirow{2}{*}{ Físicos, químicos y asimilados } \\
\hline 261 & \\
\hline 202 & \multirow{2}{*}{ Matemáticos, actuarios, estadísticos y asimilados } \\
\hline 262 & \\
\hline 203 & \multirow{2}{*}{ Profesionales de la informática de nivel superior } \\
\hline 263 & \\
\hline 204 & \multirow{2}{*}{ Arquitectos, urbanistas e ingenieros de planificación del tráfico } \\
\hline 264 & \\
\hline 205 & \multirow{2}{*}{ Ingenieros superiores y técnicos } \\
\hline 265 & \\
\hline 231 & Abogados y fiscales \\
\hline 232 & Jueces y magistrados \\
\hline 239 & Otros profesionales del derecho \\
\hline 241 & \multirow{2}{*}{ Profesionales en administración y dirección de empresas } \\
\hline 291 & \\
\hline 252 & \multirow{2}{*}{ Archiveros, bibliotecarios y profesionales asimilados } \\
\hline 292 & \\
\hline 303 & Profesionales técnicos de la informática \\
\hline 304 & Operadores de equipos ópticos y electrónicos \\
\hline 331 & Profesionales de apoyo en operaciones financieras y comerciales \\
\hline 332 & Representantes de comercio y técnicos de venta \\
\hline 341 & $\begin{array}{c}\text { Profesionales de apoyo a la gestión administrativa con tareas administrativas } \\
\text { generales }\end{array}$ \\
\hline 351 & Consignatarios y agentes en la contratación de mano de obra \\
\hline 401 & Auxiliares contables y financieros \\
\hline 421 & Taquígrafos y mecanógrafos \\
\hline 422 & Grabadores de datos \\
\hline
\end{tabular}


A.1 (cont.). Clasificación de empleo TIC por ocupaciones. (Fuente: $\mathrm{CNO}$ - 94)

\begin{tabular}{|c|c|}
\hline 430 & Auxiliares administrativos sin tareas de atención al público \\
\hline 440 & Auxiliares administrativos con tareas de atención al público \\
\hline 762 & Mecánicos y ajustadores de equipos eléctricos y electrónicos \\
\hline
\end{tabular}

A.2. Ocupaciones TIC masculinizadas en los años 2002 y 2007. (Fuente: elaboración propia a partir de la EPA)

\begin{tabular}{|c|c|}
\hline 2002 & 2007 \\
\hline Dirección general y presidencia ejecutiva & Dirección general y presidencia ejecutiva \\
\hline Dirección de departamento de producción & Dirección de departamento de producción \\
\hline $\begin{array}{l}\text { Dirección de áreas y departamentos } \\
\text { especializados }\end{array}$ & $\begin{array}{l}\text { Dirección de áreas y departamentos } \\
\text { especializados }\end{array}$ \\
\hline $\begin{array}{l}\text { Profesionales de la informática de nivel } \\
\text { superior }\end{array}$ & $\begin{array}{l}\text { Profesionales de la informática de nivel } \\
\text { superior }\end{array}$ \\
\hline $\begin{array}{l}\text { Arquitectos, urbanistas e ingenieros } \\
\text { planificadores de tráfico }\end{array}$ & $\begin{array}{l}\text { Arquitectos, urbanistas e ingenieros } \\
\text { planificadores de tráfico }\end{array}$ \\
\hline Ingenieros superiores & Ingenieros superiores \\
\hline \multicolumn{2}{|l|}{ Jueces y magistrados } \\
\hline Físicos, químicos y asimilados & Físicos, químicos y asimilados \\
\hline $\begin{array}{c}\text { Matemáticos, actuarios, estadísticos y } \\
\text { asimilados }\end{array}$ & $\begin{array}{c}\text { Matemáticos, actuarios, estadísticos y } \\
\text { asimilados }\end{array}$ \\
\hline $\begin{array}{l}\text { Profesionales de nivel medio de la } \\
\text { informática }\end{array}$ & $\begin{array}{l}\text { Profesionales de nivel medio de la } \\
\text { informática }\end{array}$ \\
\hline Arquitectos técnicos & Arquitectos técnicos \\
\hline Ingenieros técnicos & Ingenieros técnicos \\
\hline Profesionales técnicos de la informática & Profesionales técnicos de la informática \\
\hline Operadores de equipos ópticos y electrónicos & Operadores de equipos ópticos y electrónicos \\
\hline $\begin{array}{l}\text { Representantes de comercio y técnicos de } \\
\text { venta }\end{array}$ & $\begin{array}{l}\text { Representantes de comercio y técnicos de } \\
\text { venta }\end{array}$ \\
\hline \multicolumn{2}{|l|}{$\begin{array}{c}\text { Consignatarios y agentes en la contratación } \\
\text { de mano de obra }\end{array}$} \\
\hline $\begin{array}{l}\text { Mecánicos y ajustadores de equipos } \\
\text { eléctricos y electrónicos }\end{array}$ & \\
\hline
\end{tabular}


A.3. Ocupaciones TIC feminizadas en los años 2002 y 2007. (Fuente: elaboración propia a partir de la EPA)

\begin{tabular}{|c|c|}
\hline \multicolumn{1}{|c|}{2002} & 2007 \\
\hline $\begin{array}{c}\text { Matemáticos, actuarios, estadísticos y asimi- } \\
\text { lados }\end{array}$ & $\begin{array}{c}\text { Matemáticos, actuarios, estadísticos y asimi- } \\
\text { lados }\end{array}$ \\
\hline $\begin{array}{c}\text { Archiveros, bibliotecarios y profesionales } \\
\text { asimilados }\end{array}$ & $\begin{array}{c}\text { Archiveros, bibliotecarios y profesionales } \\
\text { asimilados }\end{array}$ \\
\hline $\begin{array}{l}\text { Diplomados en contabilidad y graduados } \\
\text { sociales y técnicos de empresas y actividades } \\
\text { turísticas }\end{array}$ & $\begin{array}{c}\text { Diplomados en contabilidad y graduados } \\
\text { sociales y técnicos de empresas y actividades } \\
\text { turísticas }\end{array}$ \\
\hline Ayudantes de archivo, biblioteca y asimilados & Ayudantes de archivo, biblioteca y asimilados \\
\hline $\begin{array}{c}\text { Profesionales de apoyo a la gestión adminis- } \\
\text { trativa, con tareas administrativas generales }\end{array}$ & $\begin{array}{c}\text { Profesionales de apoyo a la gestión adminis- } \\
\text { trativa, con tareas administrativas generales }\end{array}$ \\
\hline Taquígrafos y mecanógrafos & Taquígrafos y mecanógrafos \\
\hline $\begin{array}{c}\text { Auxiliares administrativos sin tareas de aten- } \\
\text { ción al público no clasificados anteriormente }\end{array}$ & $\begin{array}{c}\text { Auxiliares administrativos sin tareas de aten- } \\
\text { ción al público no clasificados anteriormente }\end{array}$ \\
\hline $\begin{array}{c}\text { Auxiliares administrativos con tareas de aten- } \\
\text { ción al público no clasificados anteriormente }\end{array}$ & $\begin{array}{c}\text { Auxiliares administrativos con tareas de aten- } \\
\text { ción al público no clasificados anteriormente }\end{array}$ \\
\hline
\end{tabular}

A.4. Clasificación de empleo TIC por sectores de actividad. (Fuente: CNAE - 93)

\begin{tabular}{|c|c|}
\hline 300 & Fabricación de máquinas de oficina, ordenadores y otros equipos informáticos \\
\hline 313 & Fabricación de hilos y cables eléctricos aislados \\
\hline 321 & Fabricación de válvulas, tubos y otros componentes electrónicos \\
\hline 322 & $\begin{array}{r}\text { Fabricación de transmisores de radiodifusión y televisión y de aparatos para la } \\
\text { radiotelefonía y radiotelegrafía con hilos }\end{array}$ \\
\hline 323 & Fabricación de aparatos de recepción, grabación y reproducción de sonido e imagen \\
\hline 332 & $\begin{array}{r}\text { Fabricación de instrumentos y aparatos de medida, verificación, control, navegación y } \\
\text { otros fines, excepto equipos de control para procesos industriales }\end{array}$ \\
\hline 333 & Fabricación de equipo de control de procesos industriales \\
\hline 516 & $\begin{array}{r}\text { Comercio al por mayor de otros componentes y equipos electrónicos, ordenadores, } \\
\text { periféricos y programas informáticos }\end{array}$ \\
\hline 642 & Telecomunicaciones \\
\hline 713 & Alquiler de máquinas y equipo de oficina (incluido ordenadores) \\
\hline 721 & Consulta de equipo informático \\
\hline 722 & $\begin{array}{c}\text { Edición de programas informáticos, otras actividades de consultoría y suministro de } \\
\text { programas informáticos }\end{array}$ \\
\hline 723 & Proceso de datos \\
\hline
\end{tabular}


A.4 (cont.). Clasificación de empleo TIC por sectores de actividad. (Fuente: CNAE - 93)

\begin{tabular}{|c|c|}
\hline 724 & Actividades relacionadas con bases de datos \\
\hline 725 & $\begin{array}{c}\text { Mantenimiento y reparación de máquinas de oficina, contabilidad y equipo } \\
\text { informático }\end{array}$ \\
\hline 726 & Otras actividades relacionadas con la informática \\
\hline
\end{tabular}

